\title{
Early-Onset Bilateral Severe Valvular Regurgitation After Mediastinal Radiotherapy in Hodgkin Lymphoma Survivors: Should We Screen Prior to 10 Years After Mediastinal Radiotherapy?
}

\author{
Tushar Mishra, MD, ${ }^{1}$ Abdelrahman Ahmed, MD, ${ }^{1}$ Mohamed Shokr, MD, ${ }^{2}$ Ahmed Rashed, MD, ${ }^{2}$ Ahmed S. Yassin, MD, ${ }^{1}$ \\ Anupama Kottam, MD' \\ ${ }^{1}$ Department of Internal Medicine, Wayne State University/Detroit Medical Center, Detroit, MI ${ }^{2}$ Division of Cardiology, Wayne State \\ University/Detroit Medical Center, Detroit, MI
}

Background: Radiation-induced valvulopathy (RIV) is a common complication of mediastinal radiotherapy and usually occurs at least 10 years after exposure to radiotherapy.

Case Report: We report the case of a 37-year-old female with a history of stage IIIB Hodgkin lymphoma who was diagnosed with RIV after all other potential causes of shortness of breath and valvular dysfunction were excluded. The patient's presentation, 6 years after receiving chemotherapy and radiotherapy for Hodgkin lymphoma, was earlier than expected after mediastinal radiotherapy. The patient was started on a regimen of lisinopril, nifedipine, and metoprolol, and her symptoms improved significantly within 4 days of starting medical therapy. We review the literature, discuss the risk factors and determinants of developing RIV, and suggest the ideal timing to screen patients.

Conclusion: This case is of educational value for internal medicine, oncology, and cardiology healthcare providers who should consider RIV as a cause of shortness of breath in patients who underwent mediastinal radiotherapy for Hodgkin lymphoma.

Keywords: Heart failure, heart valve diseases, Hodgkin disease, mitral valve insufficiency, radiotherapy, tricuspid valve insufficiency

Address correspondence to Abdelrahman Ahmed, MD, Department of Internal Medicine, Wayne State University/Detroit Medical Center, 4201 St. Antoine, Detroit, MI 48201. Tel: (517) 505-8340. Email: amahmed@med.wayne.edu

\section{INTRODUCTION}

Hodgkin lymphoma is a common malignancy, especially in young adults, accounting for approximately 8,220 new cases diagnosed in the United States annually. ${ }^{1}$ Since the introduction of chemotherapy and radiotherapy, patients with Hodgkin lymphoma have improved cure rates and prognosis compared to the era preceding the introduction of these interventions, with more than $80 \%$ of patients now achieving a 20-year survival. ${ }^{2}$

Because of the increased life expectancy of these patients, healthcare providers are witnessing an increasing number of long-term side effects of these treatments. At long-term follow-up of patients after radiotherapy for Hodgkin lymphoma, cardiovascular disease was the third most common cause of death, responsible for $12 \%-16 \%$ of deaths. ${ }^{3}$ Mediastinal radiotherapy is known to cause valvular disease, pericarditis, coronary artery disease, and cardiomyopathy. ${ }^{4}$

We present the case of a patient who developed radiation-induced valvulopathy (RIV) 6 years after receiving chemotherapy and radiotherapy for Hodgkin lymphoma.

\section{CASE REPORT}

A 37-year-old female with a history of stage IIIB Hodgkin lymphoma presented with shortness of breath for 3 months. Her symptoms had gradually worsened to the extent that she could no longer walk one block without becoming short of breath. She had a cough productive of small amounts of clear sputum with occasional streaks of blood. She denied orthopnea or paroxysmal nocturnal dyspnea. Her blood pressure was 129/85 mmHg, heart rate $98 \mathrm{bpm}$, respiratory rate 17 breaths per minute, and oxygen saturation $98 \%$. Her temperature was normal. Cardiovascular examination revealed a grade 3 apical holosystolic murmur radiating to the axilla, a loud holosystolic murmur at the left lower sternal border increasing in intensity with inspiration, and bilateral lower extremity pitting edema. Six years prior, she had received 6 cycles of chemotherapy with doxorubicin (a total of $300 \mathrm{mg} / \mathrm{m}^{2}$ dose), bleomycin, vincristine, and dacarbazine. Chemotherapy was followed by radiotherapy to her mediastinum and supraclavicular fossae for a total dose of 36 Gy in a period of 30 days. 
Chest $\mathrm{x}$-ray revealed straightening of the left heart border with pulmonary vascular congestion and an $\mathrm{N}$-terminal pro b-type natriuretic peptide (NT-proBNP) level of 1,640 pg/mL (reference value, $<450 \mathrm{pg} / \mathrm{mL}$ ). Transthoracic echocardiography (TTE) demonstrated an ejection fraction of $60 \%$ and severe mitral and tricuspid valve regurgitation. The right ventricle was severely dilated and hypokinetic. Right ventricular systolic pressure was estimated to be $74 \mathrm{mmHg}$. Computed tomography (CT) imaging of the chest ruled out interstitial lung changes or the presence of pulmonary emboli that could explain the dilated right ventricle. Ventilation-perfusion (V/Q) scan was negative for chronic thromboemboli. Transesophageal echocardiogram demonstrated thickened and fibrotic atrioventricular valves with resultant malcoaptation and severe valvular regurgitation. Left heart catheterization did not reveal any coronary artery disease. Right heart catheterization revealed elevated mean pulmonary artery pressure $(60 \mathrm{mmHg})$ and pulmonary vascular resistance of 7.7 Wood units. Pulmonary capillary wedge pressure was $33 \mathrm{mmHg}$. Bilateral atrial tracings demonstrated prominent $\checkmark$ waves corresponding to severe mitral and tricuspid regurgitation.

Suspicion of pulmonary or cardiac causes in this patient was high given her history of chemotherapy and radiotherapy. Both bleomycin and radiotherapy can cause pulmonary fibrosis and subsequent shortness of breath. 5,6 However, the findings of heart murmurs on physical examination and elevated NT-proBNP suggested a cardiac cause. Constrictive pericarditis and restrictive cardiomyopathy were in the differential given the patient's history of radiotherapy. ${ }^{7}$ Cardiomyopathy as a result of chemotherapy or radiotherapy was also being considered. The possibility of RIV was initially low given that most clinically significant symptomatic cases are reported at least 10 years after radiotherapy, which was not the case for our patient. ${ }^{7}$ Given the TTE findings of bilateral severe mitral and tricuspid regurgitation, our workup aimed to clarify the cause of the valvulopathy. The patient's left ventricular ejection fraction was normal, so functional mitral regurgitation was unlikely. TTE also revealed a severely dilated right ventricle, indicating that cor pulmonale might be the cause. However, a normal high-resolution CT scan and pulmonary function test ruled out interstitial lung disease. In addition, the V/Q scan did not reveal any ventilation/perfusion mismatch suggestive of chronic pulmonary thromboembolic disease. Simultaneous left and right heart catheterization did not reveal any interventricular dependence as would be seen in constrictive or restrictive cardiomyopathy. No evidence of atherosclerotic disease was found on coronary angiography. The nonspecific valve thickening and severe regurgitation of mitral and tricuspid valves suggested primary valve disease.

After all the potential causes of shortness of breath and valvular dysfunction were excluded, the diagnosis of RIV was made. The patient was started on a regimen of lisinopril, nifedipine, and metoprolol, and her symptoms improved significantly within 4 days of starting medical therapy. Because the primary pathology of RIV damage cannot be reversed with medication therapy, the patient was referred to cardiothoracic surgery for further management. This patient also possibly had pulmonary arterial hypertension $(\mathrm{PAH})$ that can partly explain the severe tricuspid regurgitation. Studies have demonstrated that $\mathrm{PAH}$ can occur after radiotherapy or chemotherapy. 8,9

\section{DISCUSSION}

RIV should always be considered as a cause of dyspnea in patients who received radiotherapy in the past. Diagnosis can be challenging because many other causes, pulmonary and cardiac, can affect the integrity of the heart valves and result in valvular dysfunction. Echocardiography may not reveal an underlying cause for the regurgitation. Many factors interact to determine the extent of valvular damage in these patients, making it difficult to establish the best time to start screening for this condition.

The prevalence of RIV reported in the literature varies widely, from $2 \%-37 \%$ in Hodgkin lymphoma survivors after mediastinal radiotherapy, which is much higher than the prevalence of valvular heart disease in the general US population, reported to be approximately $2.5 \%$ in a large pooled population-based study. ${ }^{10,11}$ This wide variation of prevalence in RIV can be attributed to the different study designs and diagnostic techniques used to assess patients with Hodgkin lymphoma. ${ }^{11}$ Many investigators reported an incidence of RIV of approximately $4 \%$ and a median interval to diagnosis ranging from $18-23$ years. ${ }^{12}$ Mediastinal radiotherapy increases the risk of valvular heart disease by 2- to $12-$ fold. ${ }^{13,14}$

The aortic valve is the most commonly affected valve, followed by the mitral and tricuspid valves. ${ }^{7}$ The most common lesions reported are aortic stenosis followed by mitral regurgitation, tricuspid regurgitation, and mitral stenosis. ${ }^{12}$ The increasing involvement of the aortic valve is thought to be attributable to the proximity of the valve to the mediastinal radiation field. ${ }^{7}$ However, our patient had involvement of both mitral and tricuspid valves with features of severe regurgitation without the involvement of the aortic valve.

The mechanism of valvulopathy with radiation is not fully understood. Because no blood vessels are in the valves, the pathophysiology appears to be different from that of coronary artery disease. The more frequent involvement of left-sided valves suggests that higher pressures act as a direct contributor or as a trigger for initiating the damage that continues and accumulates for many years, leading to RIV. Radiation induces the inflammatory, mesenchymal, and epithelial cells to produce transforming growth factor beta-1 (TGF- $\beta 1$ ), which activates fibroblasts into matrixproducing myofibroblasts, leading to collagen formation and fibrosis. ${ }^{15}$ After initiation, TGF- $\beta 1$ is no longer required to sustain the process, as fibrosis is further perpetuated by autocrine induction of a cytokine called connective tissue growth factor. Twenty years after the inciting event, the process of valve damage slowly progresses, leading to clinically significant valvulopathy. Another suggestion is that radiation activates an osteogenic phenotype in human aortic valve interstitial cells, leading to increased production of osteogenic factors such as bone morphogenetic protein 2 , osteopontin, alkaline phosphatase, and transcription factor RUNX2, which would explain the increased calcification of valves seen in RIV. ${ }^{16}$

Risk factors for the development of RIV include dose of radiation, time since treatment, adjuvant anthracycline chemotherapy, and age at presentation. ${ }^{12-14}$ Understanding 
the interplay among these factors can help predict the risk of RIV in individual patients. A retrospective study of 1,852 Hodgkin lymphoma survivors found a nonlinear relationship between radiation dose and the incidence of RIV. The risk of RIV at doses $<30$ Gy was very low, but the risk increased incrementally with doses $>30$ Gy. For exposure to radiation doses of 31-35 Gy, 36-40 Gy, and >40 Gy, the risk of RIV increased by factors of $3.1 \%, 5.45 \%$, and $11.8 \%$, respectively. ${ }^{13}$ Other studies have also reported a mean dose of 30-46 Gy associated with the development of RIV. ${ }^{1,7,17}$ In the 1960s, patients with Hodgkin lymphoma received a total mantle field radiation of 35-45 Gy spreading to lymph nodes in the neck, mediastinum, and axillae, resulting overall in 27.5 Gy to the heart and $>35$ Gy to parts of the heart such as the pulmonary and aortic valves. ${ }^{3,18}$ In 2004, involved-field radiotherapy, which requires a mean dose of $30 \mathrm{~Gy}$, became the gold standard for early-stage Hodgkin lymphoma. ${ }^{19}$ Our patient had stage IIIB Hodgkin lymphoma and received a total dose of $36 \mathrm{~Gy}$, so she was clearly at risk of RIV.

The increasing prevalence of RIV with time has been reported in multiple studies. Heidenreich et al studied 294 Hodgkin lymphoma survivors and demonstrated an increased risk of aortic regurgitation, tricuspid regurgitation, and aortic stenosis 20 years after mediastinal radiotherapy compared to patients who had mediastinal radiotherapy less than 10 years before evaluation. ${ }^{7}$ In a retrospective study of 415 patients with Hodgkin lymphoma who underwent mediastinal radiotherapy, the incidence of clinically significant valvular dysfunction 10 years after treatment was $1 \%$ and the incidence at 20 years was $6 \% .^{20}$ The median duration for development of clinically significant valvular dysfunction was 22 years. Findings from both of these studies were in agreement that the risk of developing RIV within 10 years of treatment is relatively low. Our patient developed features of RIV within 6 years of receiving treatment, which is not common.

The adjuvant use of anthracyclines as part of chemotherapy has been reported to double the incidence of valvular dysfunction in Hodgkin lymphoma survivors, while a nonanthracycline-based regimen did not increase the risk. ${ }^{14}$ van Nimwegan et al demonstrated an incremental incidence of valvular disease with increasing doses of an anthracycline-based regimen. ${ }^{21}$ The hazard ratios for developing valvular heart disease were 1.1, 2.2, and 3.7 for patients who received dose ranges of $35-200 \mathrm{mg} / \mathrm{m}^{2}, 210-$ $325 \mathrm{mg} / \mathrm{m}^{2}$, and $350-880 \mathrm{mg} / \mathrm{m}^{2}$, respectively. Our patient received a dose of $300 \mathrm{mg} / \mathrm{m}^{2}$ and thus had increased chances of developing RIV.

Given the interplay among these factors and the risk of RIV, formulating a predictive model would be constructive, taking into account the most common variables such as radiation dose, time since treatment, and adjuvant anthracycline dose to develop an objective risk assessment for each patient. Then the screening period could be defined depending on patient-specific risks. Cella et al have already attempted to use dosimetric predictors and multiple variables to predict the risk of asymptomatic heart disease following mediastinal radiation in Hodgkin lymphoma survivors. ${ }^{22,23}$ These prediction models have shown high agreement between observed and predicted outcomes, but the major challenge for their use is that many factors that have not been accounted for might affect the risk of developing RIV. Also, more external validation must be performed before these models can be applied in clinical practice.

Although the evidence from prospective trials is lacking, multiple retrospective studies have recommended screening Hodgkin lymphoma survivors who have received radiotherapy beginning 10 years after treatment. ${ }^{1,3,7}$ Echocardiography is the initial diagnostic modality, and cardiac magnetic resonance imaging can provide complementary information, including identifying myocardial fibrosis. ${ }^{11}$ Echocardiographic evidence of valve thickening may be present earlier than the actual dysfunction, thus providing a window of opportunity for screening. The characteristic pattern of thickening of aortic and mitral valves involving aortic-mitral curtains has been reported in the literature. ${ }^{24}$ Echocardiographic evidence of aortic-mitral calcification was present in $39 \%$ patients at 5 years and in approximately $90 \%$ of patients at 20 years. ${ }^{7}$ Our patient was only 6 years posttreatment, but she developed features of severe valvular dysfunction that were probably attributable to the higher radiation dose requirement and concomitant anthracycline use. Therefore, in addition to time since radiotherapy, taking other factors, such as the dose of radiation and concomitant anthracycline use, into account is crucial when deciding the ideal screening interval for each patient. Management of these patients usually includes either surgical or percutaneous modalities, and the optimal choice is still not clearly defined. A study of 22 patients who had RIV and underwent valve repair showed no difference in 5-year survival or freedom from reoperation or transplantation compared to a cohort who underwent valve replacement. ${ }^{25}$ In a study of 37 patients who underwent surgery, transcatheter aortic valve implantation (TAVI), or transcatheter mitral valve implantation (TMVI), the transcatheter groups had a lower overall inhospital mortality (25\% in the surgery group, $6 \%$ in the TAVI group, and $0 \%$ in the TMVI group) and shorter lengths of stay (14 days in the surgery group, 7 days in the TAVI group, and 6 days in the TMVI group). ${ }^{26}$ One-year mortality was $25 \%$ in the surgery group, $18 \%$ in the TAVI group, and $50 \%$ in the TMVI group, with overall mortality of $24 \%$. Wu et al compared outcomes of open heart surgery in patients with previous breast cancer or Hodgkin lymphoma who had a confirmed diagnosis of RIV and/or radiation-induced heart disease to a group of controls without previous radiation or malignancy. ${ }^{27}$ After a mean follow-up period of 7.6 years, the mortality rate was higher in the radiation group vs the control group (55\% vs $28 \%, P<0.001$ ).

The generalizability of these studies is limited by their small sample sizes and the differences in underlying severity of disease in the patients, thus confounding the results and interpretability of these studies. A transcatheter approach (TAVI or TMVI) can be considered in patients with RIV given the high likelihood of the presence of mediastinal fibrosis, chest wall deformities, and porcelain aorta that can make surgical approach difficult.

\section{CONCLUSION}

As the life expectancy of patients with Hodgkin lymphoma has substantially improved, RIV is becoming an increasingly recognized disease entity. Our patient's presentation was unique because she presented relatively early after treatment completion with severe mitral and tricuspid dysfunction that are rarely involved in combination and without the 
involvement of the aortic valve. The recommended screening duration of 10 years might not be applicable to all patient groups, and other risk factors such as radiation dose and concomitant anthracycline use-along with the application of predictive models-should be used when deciding the optimal timing for screening each patient.

\section{ACKNOWLEDGMENTS}

The authors have no financial or proprietary interest in the subject matter of this article.

\section{REFERENCES}

1. Bijl JM, Roos MM, van Leeuwen-Segarceanu EM, et al. Assessment of valvular disorders in survivors of Hodgkin's lymphoma treated by mediastinal radiotherapy \pm chemotherapy. Am J Cardiol. 2016 Feb 15;117(4):691-696. doi: 10.1016/j.amjcard.2015.11.027.

2. Brenner $\mathrm{H}$, Gondos A, Pulte D. Ongoing improvement in long-term survival of patients with Hodgkin disease at all ages and recent catch-up of older patients. Blood. 2008 Mar 15;111(6):2977-2983.

3. Lee MS, Finch W, Mahmud E. Cardiovascular complications of radiotherapy. Am J Cardiol. 2013 Nov 15;112(10):1688-1696. doi: 10.1016/j.amjcard.2013.07.031.

4. Carlson RG, Mayfield WR, Normann S, Alexander JA. Radiation-associated valvular disease. Chest. 1991 Mar;99(3):538-545.

5. Simone CB 2nd. Thoracic radiation normal tissue injury. Semin Radiat Oncol. 2017 Oct;27(4):370-377. doi: 10.1016/j.semradonc.2017.04.009.

6. Madabhavi I, Modi G, Patel A, Anand A, Panchal H, Parikh S. Pulmonary toxicity following bleomycin use: a single-center experience. J Cancer Res Ther. 2017 Jul-Sep;13(3):466-470. doi: 10.4103/0973-1482.204887.

7. Heidenreich PA, Hancock SL, Lee BK, Mariscal CS, Schnittger I. Asymptomatic cardiac disease following mediastinal irradiation. J Am Coll Cardiol. 2003 Aug 20;42(4): 743-749.

8. Perkett EA, Brigham KL, Meyrick B. Increased vasoreactivity and chronic pulmonary hypertension following thoracic irradiation in sheep. J Appl Physiol (1985). 1986 Nov;61(5):1875-1881.

9. Ranchoux B, Günther S, Quarck R, et al. Chemotherapy-induced pulmonary hypertension: role of alkylating agents. Am J Pathol. 2015 Feb;185(2):356-371. doi: 10.1016/j.ajpath.2014.10.021.

10. Nkomo VT, Gardin JM, Skelton TN, Gottdiener JS, Scott CG, Enriquez-Sarano M. Burden of valvular heart diseases: a population-based study. Lancet. $2006 \mathrm{Sep}$ 16;368(9540):1005-1011.

11. Gujral DM, Lloyd G, Bhattacharyya S. Radiation-induced valvular heart disease. Heart. 2016 Feb 15;102(4):269-276. doi: 10.1136/heartjnl-2015-308765.

12. Hull MC, Morris CG, Pepine CJ, Mendenhall NP. Valvular dysfunction and carotid, subclavian, and coronary artery disease in survivors of hodgkin lymphoma treated with radiation therapy. JAMA. $2003 \mathrm{Dec}$ 3;290(21):2831-2837.
13. Cutter DJ, Schaapveld M, Darby SC, et al. Risk of valvular heart disease after treatment for Hodgkin lymphoma. J Natl Cancer Inst. 2015 Feb 23;107(4). doi: 10.1093/jnci/djv008.

14. Aleman BM, van den Belt-Dusebout AW, De Bruin ML, et al. Late cardiotoxicity after treatment for Hodgkin lymphoma. Blood. 2007 Mar 1;109(5):1878-1886.

15. Yarnold J, Brotons MC. Pathogenetic mechanisms in radiation fibrosis. Radiother Oncol. 2010 Oct;97(1):149-161. doi: 10.1016/j.radonc.2010.09.002.

16. Nadlonek NA, Weyant MJ, Yu JA, et al. Radiation induces osteogenesis in human aortic valve interstitial cells. J Thorac Cardiovasc Surg. 2012 Dec;144(6):1466-1470. doi: 10.1016/j.jtcvs.2012.08.041.

17. Veinot JP, Edwards WD. Pathology of radiation-induced heart disease: a surgical and autopsy study of 27 cases. Hum Pathol. 1996 Aug;27(8):766-773.

18. Hodgson DC. Late effects in the era of modern therapy for Hodgkin lymphoma. Hematology Am Soc Hematol Educ Program. 2011;2011:323-329. doi: 10.1182/asheducation-2011.1.323.

19. Witkowska M, Majchrzak A, Smolewski P. The role of radiotherapy in Hodgkin's lymphoma: what has been achieved during the last 50 years? Biomed Res Int. 2015;2015:485071. doi: 10.1155/2015/485071.

20. Hull MC, Morris CG, Pepine CJ, Mendenhall NP. Valvular dysfunction and carotid, subclavian, and coronary artery disease in survivors of hodgkin lymphoma treated with radiation therapy. JAMA. $2003 \mathrm{Dec}$ 3;290(21):2831-2837.

21. van Nimwegen $F A$, Schaapveld $M$, Janus $C P$, et al. Cardiovascular disease after Hodgkin lymphoma treatment: 40-year disease risk. JAMA Intern Med. 2015 Jun;175(6):1007-1017. doi: 10.1001/jamainternmed.2015.1180.

22. Cella L, Liuzzi R, Conson M, et al. Dosimetric predictors of asymptomatic heart valvular dysfunction following mediastinal irradiation for Hodgkin's lymphoma. Radiother Oncol. 2011 Nov;101(2):316-321. doi: 10.1016/j.radonc.2011.08.040.

23. Cella L, Oh JH, Deasy JO, et al. Predicting radiation-induced valvular heart damage. Acta Oncol. 2015 Nov;54(10):1796-1804. doi: 10.3109/0284186X.2015.1016624.

24. Brand MD, Abadi CA, Aurigemma GP, Dauerman HL, Meyer TE. Radiation-associated valvular heart disease in Hodgkin's disease is associated with characteristic thickening and fibrosis of the aortic-mitral curtain. $J$ Heart Valve Dis. 2001;10(5):681-685.

25. Crestanello JA, McGregor CG, Danielson GK, et al. Mitral and tricuspid valve repair in patients with previous mediastinal radiation therapy. Ann Thorac Surg. 2004 Sep;78(3):826-831; discussion 826-831.

26. Paven E, Cimadevilla C, Urena M, et al. Management of radiation-induced valvular heart disease due to Hodgkin's Lymphoma in the modern area. Eurolntervention. 2018 Feb 2;13(15):e1771-e1773. doi: 10.4244/EIJ-D-17-00811.

27. Wu W, Masri A, Popovic ZB, et al. Long-term survival of patients with radiation heart disease undergoing cardiac surgery: $a$ cohort study. Circulation. 2013 Apr 9;127(14):1476-1485. doi: 10.1161/CIRCULATIONAHA.113.001435.

This article meets the Accreditation Council for Graduate Medical Education and the American Board of Medical Specialties Maintenance of Certification competencies for Patient Care and Medical Knowledge. 